\title{
Un télémètre transportable de résolution submicrométrique
}

\section{A sub-micrometric resolution transportable telemeter}

\author{
Shéhérazade AZOUIGUI ${ }^{1}$, Thomas BADR ${ }^{1,2}$, Patrick JUNCAR $^{1}$, Marc HIMBERT ${ }^{1}$ \\ et Jean-Pierre WALLERAND ${ }^{1}$ \\ 1 Laboratoire commun de métrologie LNE-CNAM (LCM), LNE, 1 rue Gaston Boissier, 75015 Paris, France, jean-pierre.wallerand@ cnam.fr. \\ 2 Adresse actuelle : Laboratoire de Physique des Lasers, Université Paris 13 Nord (UP13), 99, Av. J.B. Clément, 93430 Villetaneuse, France.
}

\section{Résumé}

Nous décrivons dans cet article la réalisation d'un télémètre transportable basé sur un principe d'interférométrie à longueur d'onde synthétique. Les sources lasers utilisées sont deux lasers YAG à $532 \mathrm{~nm}$ doublés en fréquence. Une technique de détection superhétérodyne a été mise en place pour réaliser une mesure de phase à $2 \pi / 5600$ près. Une exactitude d'environ $5 \mu \mathrm{m}(1 \sigma)$ a été mesurée sur $25 \mathrm{~m}$, par comparaison avec un banc de déplacement interférométrique. Le système a été transporté en Finlande (Nummela) pour mesurer une ligne géodésique de référence, avec une incertitude type de $700 \mu \mathrm{m}$. Ce travail s'est fait dans le cadre du projet européen (JRP de l'EMRP) «Long distance » qui s'est achevé en juin 2011.

MOTS CLÉS : TÉLÉMÉTRIE LASER, DÉTECTION SUPERHÉTÉRODYNE, INTERFÉROMÉTRIE, GÉODÉSIE.

\begin{abstract}
This paper describes a transportable system based on synthetic wavelength interferometry for absolute distance measurement. The synthetic wavelength is generated by means of two frequency-doubled Nd:YAG lasers. A superheterodyne technique has been implemented to detect the synthetic phase, enabling a fringe interpolation of $\sim 2 \pi / 5600$. $A \sim 5 \mu \mathrm{m}$ accuracy $(1 \sigma)$ is demonstrated over $25 \mathrm{~m}$, based on an indoor comparison with a classical fringe counting interferometer. An outdoor comparison has been carried out on a 864 m-long standard baseline located in Finland (Nummela), yielding an uncertainty of $700 \mu \mathrm{m}(1 \sigma)$. This work was carried out within the framework of the "Long distance" EMRP project that ended on June 2011.
\end{abstract}

KEY WORDS: LASER TELEMETRY, SUPERHETERODYNE DETECTION, INTERFEROMETRY, GEODESY.

\section{Introduction}

La géodésie et la topographie sont les disciplines qui ont motivé le développement de systèmes de mesure de grandes distances ces derniers siècles. Pour mémoire, la toute première définition du mètre a été adoptée après un travail monumental de triangulation entre Dunkerque et Barcelone à la fin du $18^{\mathrm{e}}$ siècle [1]. Depuis, des systèmes de mesure de plus en plus sophistiqués ont été développés pour répondre aux besoins de mesure de grandes dimensions : réception satellite (GPS par exemple) pour l'automatisation ou le positionnement de machines (en agriculture notamment) et la topographie (travail de topographie en souterrains, réseaux de capteurs pour la prévention de catastrophes, ou la maintenance des échelles de référence à l'échelle planétaire). On peut citer pour exemple l'expérience de mesure de temps de vol de neutrinos entre le CERN et le détecteur OPERA dans le laboratoire souterrain de Gran Sasso en Italie. L'incertitude finale de $20 \mathrm{~cm}$ de cette mesure de $730 \mathrm{~km}$ était essentiellement limitée par la mesure du dernier kilomètre, réalisée par un distance-mètre électro-optique (EDM) dans un tunnel [2]. La confiance dans la sureté des installations sensibles dépend également de mesures de distance fines puisqu'à proximité des centrales nucléaires, la surveillance de la déformation du sol se fait avec des résolutions submillimétriques [3].

Les grands fabricants actuels dans le domaine de la géodésie et topographie proposent des solutions affichant une exactitude meilleure que $2 \mathrm{~mm}$ sur $1 \mathrm{~km}$ [4]. 
Ces appareils sont qualifiés selon la norme ISO17123-4 qui est un simple guide de vérification de ces instruments basé sur l'estimation de leur répétabilité [5]. La longueur exacte des lignes géodésiques utilisées pour ces vérifications n'a pas besoin d'être connue. Certaines lignes géodésiques sont toutefois traçables au mètre le plus souvent en transférant la connaissance d'une ligne géodésique de référence [6] par l'intermédiaire d'un télémètre bien reproductible. Ces comparaisons peuvent se faire avec des incertitudes de quelques centaines de micromètres, selon les conditions atmosphériques et la qualité de la ligne géodésique mesurée [7]. Le télémètre de transfert le plus réputé est sans aucun doute le Mekometer ME5000 [8], un appareil fabriqué dans les années 1980 par la société Kern\&Co (acquis en 1988 par Leica) qui permet des mesures jusqu'à $3 \mathrm{~km}$ avec une résolution ultime de $100 \mu \mathrm{m}$. Ce télémètre n'est plus fabriqué aujourd'hui mais constitue actuellement le seul instrument de mesure suffisamment reproductible pour comparer des lignes géodésiques entre elles avec une incertitude submillimétrique.

De nombreux travaux ont été menés ces dernières années pour réaliser des télémètres innovants en vue d'améliorer l'état de l'art. Les techniques utilisées sont très variées, même si le principe de base est toujours la mesure par un moyen ou un autre du temps de propagation de la lumière. La première grande famille de techniques consiste à mesurer le déphasage d'une modulation appliquée à un faisceau lumineux, accumulé pendant la traversée de la distance à mesurer. Connaissant la vitesse de la lumière dans le milieu on déduit de ce déphasage, soumis aux variations de l'indice de l'air, la distance parcourue par la lumière. Toute la subtilité consiste ensuite à choisir de bonnes techniques pour générer la modulation de la lumière à des fréquences élevées, pour détecter cette modulation et mesurer sa phase sans générer d'erreur sur la mesure de distance. L'autre famille de techniques utilise l'interférométrie optique. Bien que cette technique soit bien connue pour obtenir des résolutions meilleures que le nanomètre [9], elle est difficilement utilisable directement pour des mesures de grandes distances sans déplacement. En effet, la phase optique mesurée donne une distance à une demi-longueur d'onde optique près et la détermination de l'ordre d'interférence peut être irréalisable si la distance à mesurer est très grande. D'autre part, les vibrations mécaniques ou les fluctuations de l'indice de l'air peuvent rendre cette technique inopérante en extérieur.

Néanmoins, pour contourner ce problème, la technique dite d'interférométrie à longueur d'onde synthétique (SWI pour «Synthetic Wavelength Interferometry») ou à deux longueurs d'onde [10] est utilisée pour mesurer des distances de plusieurs dizaines de mètres avec des incertitudes relatives de quelques $10^{-6}$. C'est cette technique que nous avons choisie d'utiliser dans le travail présenté ici. La SWI consiste à injecter, dans un interféromètre, deux longueurs d'ondes optiques, produisant un interférogramme modulé en amplitude à une longueur d'onde synthétique $\Lambda_{s}\left(=c / \Delta v_{s}\right)$ beaucoup plus grande que chacune des longueurs d'ondes optiques. Cette longueur d'onde synthétique ne dépend que de la vitesse de la lumière $c$ et de la fréquence synthétique $\Delta v_{s}$, écart entre les fréquences des deux lasers éclairant l'interféromètre. Pour un écart de fréquence de $10 \mathrm{GHz}$ entre les deux lasers, la longueur d'onde synthétique est d'environ $3 \mathrm{~cm}$. La mesure de distance peut être réalisée en mesurant le déphasage de cette onde synthétique lors de sa propagation. Différentes méthodes peuvent être employées pour générer une longueur d'onde synthétique stable : une méthode compacte et relativement bon marché consiste à utiliser une source laser unique et un système de décalage de fréquence optique (un modulateur acousto-optique par exemple). Néanmoins, ceci limite la plage d'accord de la longueur d'onde synthétique. En utilisant deux sources lasers distinctes, la plage de variation de la longueur d'onde synthétique est augmentée mais une stabilisation active de la différence de fréquence entre les deux lasers est nécessaire : boucle à verrouillage de phase sur une référence hyperfréquence [11], stabilisation des lasers sur un peigne de fréquence optique (résonateur optique [12], laser femtoseconde [13, 14] ou transitions atomiques ou moléculaires [15]) ou stabilisation sur un artefact dimensionnel [16]. Nous avons fait le choix d'utiliser deux lasers YAG doublés en fréquence à $532 \mathrm{~nm}$. Un écart de fréquences de quelques gigahertz à $40 \mathrm{GHz}$ entre les deux lasers peut être obtenu, soit une longueur d'onde synthétique variant de quelques millimètres à quelques centimètres. Cet écart de fréquences est contrôlé par une boucle à verrouillage de phase sur une référence de fréquence.

\section{Description de l'instrument de mesure}

\subsection{Principe de l'interféromètre superhétérodyne à longueur d'onde synthétique}

L'interférométrie à deux longueurs d'onde ou à longueur d'onde synthétique consiste à injecter un interféromètre de type Michelson avec deux faisceaux lasers de fréquences optiques $v_{1}$ et $v_{2}$ et de mesurer la phase synthétique, c'est-à-dire la différence entre les phases optiques individuelles $\Phi_{1}$ et $\Phi_{2}$ respectivement à $v_{1}$ et $v_{2}$. Cette phase synthétique peut s'écrire sous la forme :

$$
\Phi_{s}=\Phi_{2}-\Phi_{1}=\frac{2 \pi}{\Lambda_{s}} n_{g} D
$$

où $D$ est la différence de chemin optique entre les deux bras de l'interféromètre et $n_{g}$ l'indice de groupe vu par les faisceaux, les $n_{i}$ étant les indices de phase aux fréquences optiques $v_{i}$, calculés par les formules de Ciddor $[17,18]$ :

$$
n_{g}=\frac{n_{2} v_{2}-n_{1} v_{1}}{v_{2}-v_{1}}
$$

$\Lambda_{s}$ est la longueur d'onde synthétique dans le vide, reliée à la fréquence synthétique $\Delta v_{s}$ par :

$$
\Delta v_{s}=v_{2}-v_{1}=\frac{c}{\Lambda_{s}}
$$

où $c$ est la vitesse de la lumière dans le vide. 


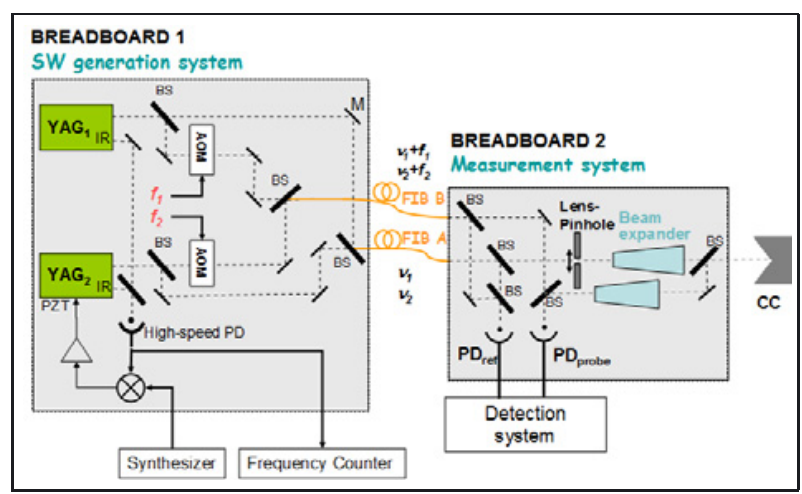

Fig. 1. - Schéma optique de l'interféromètre superhétérodyne à deux longueurs d'onde : $\mathrm{YAG}_{1,2}$, lasers YAG; IR, sortie infrarouge des lasers; AOM, modulateur acousto-optique; BS, séparateur de faisceaux; CC, Coin de cube creux; M, miroir; $\mathrm{PD}_{\text {probe }}$, photodétecteur de mesure ; $\mathrm{PD}_{\text {ref }}$, photodétecteur de référence ; PZT, céramique piezoélectrique; SW, longueur d'onde synthétique.

La phase synthétique est détectée par une technique dite superhétérodyne [19] : on module la fréquence synthétique et on détecte l'interférogramme à cette fréquence de modulation. En pratique, pour chaque faisceau de fréquence $v_{1}$ et $v_{2}$, on génère un autre faisceau légèrement décalé en fréquence respectivement d'une quantité $f_{1}$ et $f_{2}$. La détection de la phase synthétique est ensuite réalisée à la fréquence superhétérodyne $f_{2}-f_{1}$. Cette phase doit être ensuite mise sous la forme : $\Phi_{s}=$ $2 k \pi+\varphi_{s}$, autrement dit, l'ordre d'interférence synthétique $k$ doit être déterminé. Ceci se fait en réalisant la mesure de phase pour différentes valeurs de la longueur d'onde synthétique et en appliquant le principe des excédents fractionnaires.

\subsection{Réalisation expérimentale}

\subsubsection{Montage optique}

L'ADM (Absolute Distance Measurement system) du LCM est fondé sur l'utilisation de deux lasers Nd:YAG doublés en fréquence (modèle Prometheus, 20 mW, Innolight $\mathrm{GmbH}$, récemment acquis par Coherent Inc.) émettant aux alentours de $532 \mathrm{~nm}$. Grâce à l'accordabilité de chaque laser sur environ $30 \mathrm{GHz}$, nous disposons d'une longueur d'onde synthétique (écart de longueur d'onde entre les deux lasers) largement accordable. Une valeur minimale de longueur d'onde synthétique de $5 \mathrm{~mm}$ peut être obtenue lorsque les deux lasers sont séparés de $60 \mathrm{GHz}$.

Une partie de chaque laser vert est injectée dans une fibre monomode (FIB A) alors que la deuxième partie est décalée en fréquence d'une quantité $f_{1}$ pour le laser 1 et $f_{2}$ pour le laser 2 , grâce à deux modulateurs acousto-optiques avant d'être couplée dans une seconde fibre optique monomode (FIB B) (fig. 1). La fréquence superhétérodyne $f_{2}-f_{1}$ est fixée à $50 \mathrm{kHz}$. L'utilisation de fibres optiques permet une superposition parfaite des faisceaux avant l'injection dans l'interféromètre. Ce point est essentiel car un angle entre les deux faisceaux

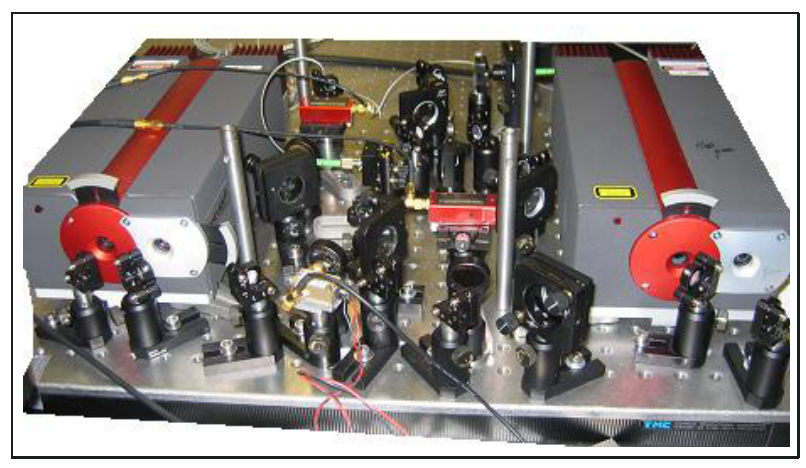

Fig. 2. - Système de génération de la longueur d'onde synthétique (breadboard 1 -dimensions : 0,45 $\mathrm{m} \times 0,60 \mathrm{~m}$ ).

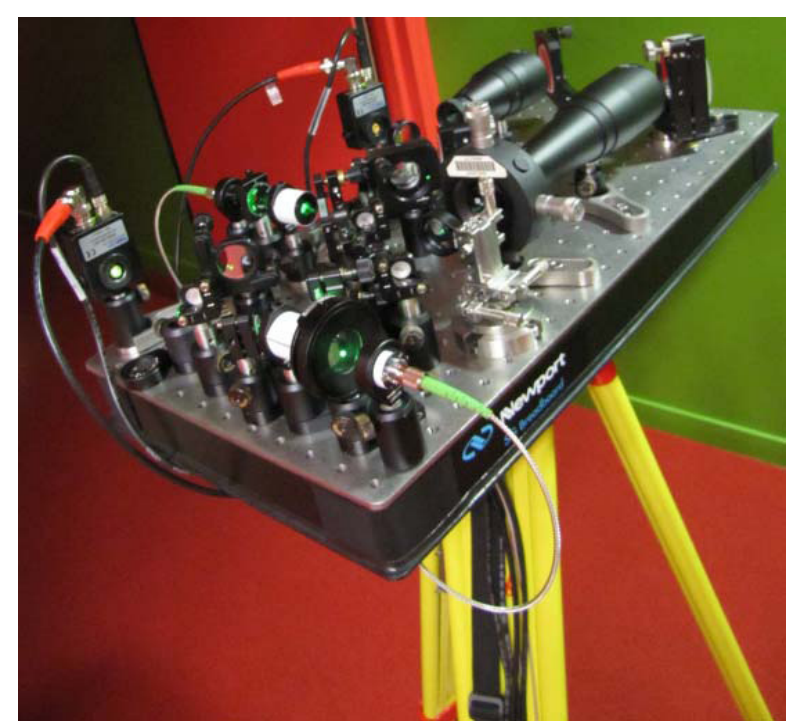

Fig. 3. - Dispositif de mesure sur un trépied (breadboard 2 dimensions : $0,3 \mathrm{~m} \times 0,6 \mathrm{~m}$ ).

induit une erreur importante sur la mesure de distance. Cela permet également de séparer physiquement la génération des longueurs d'onde synthétique (fig. 2) de la tête de mesure (fig. 3), ce qui facilite le transport du système complet [20].

Les sorties des fibres A et B sont reliées au dispositif de mesure. Le signal de mesure est le signal de battement détecté par la photodiode $\mathrm{PD}_{\text {probe }}$ entre (1) les faisceaux de fréquences $v_{i}(i=\{1 ; 2\})$ rétroréfléchis sur le coin de cube et (2) les faisceaux de fréquences $v_{i}+f_{i}$ qui arrivent directement sur la photodiode $\mathrm{PD}_{\text {probe }}$. Un signal de référence doit être également généré pour éliminer les fluctuations de phase optique parasites dues aux modulateurs acousto-optiques, aux fibres optiques ou aux chemins optiques non communs des faisceaux à $v_{1}$ et $v_{1}+f_{1}$ (respectivement $v_{2}$ et $v_{2}+f_{2}$ ). Ceci est réalisé en détectant le battement de fréquence d'une partie des faisceaux à $v_{i}$ and $v_{i}+f_{i}$ avec un autre détecteur $\left(\mathrm{PD}_{\mathrm{ref}}\right)$. La différence de phase mesurée des signaux de mesure et de référence ne dépend alors que de la différence de chemin optique vue par les faisceaux à $v_{i}+f_{i}$ et les faisceaux à $v_{i}$. 


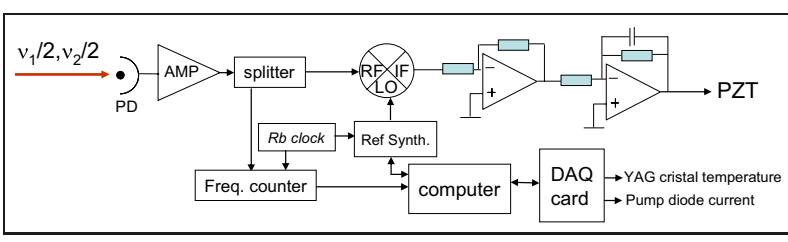

Fig. 4. - Schéma de variation de la longueur d'onde synthétique : AMP, amplificateur ; RF, radiofréquence ; LO, oscillateur local; IF, intermediate frequency; DAQ, acquisition des données; PD, photodiode infrarouge; PZT, céramique piezoélectrique.

\subsubsection{Variation de la longueur d'onde synthétique et verrouillage en phase}

La mesure absolue de distance nécessite de faire la mesure de la phase synthétique pour différentes longueurs d'ondes synthétiques (et donc fréquences synthétiques) et de comparer les différents excédents fractionnaires. Cette méthode des excédents fractionnaires est bien connue (voir par exemple la référence [21]) et est également utilisée pour déterminer l'épaisseur de cales étalons sans déplacement [22]. La mesure fine de distance est effectuée avec la fréquence synthétique la plus élevée puisque la résolution en distance est d'autant meilleure que la fréquence synthétique est élevée. Au total cinq fréquences synthétiques différentes sont utilisées pour déterminer sans ambiguiité l'ordre d'interférence correspondant à la fréquence synthétique la plus élevée. La variation de la longueur d'onde synthétique est automatisée sous Labview. Un signal de tension est d'abord envoyé par une carte d'acquisition à un des lasers pour faire varier la température du cristal laser pour atteindre approximativement la longueur d'onde synthétique requise. Une autre commande est ensuite envoyée sur le courant des diodes de pompe du laser pour un réglage fin. Enfin une boucle à verrouillage de phase permet d'asservir la différence de fréquence entre les deux lasers sur une référence de fréquence (fig. 4). Le signal d'erreur de la boucle est obtenu en détectant le battement de fréquences des sorties infrarouge des lasers à $\left(v_{2}-v_{1}\right) / 2$ grâce à une photodiode rapide (New Focus 1437, bande passante $25 \mathrm{GHz}$ ). Ce battement de fréquences est ensuite mélangé à une référence de fréquence (fixée à une valeur donnée de la longueur d'onde synthétique infrarouge) par un mélangeur de fréquence dont la sortie IF (Intermediate Frequency) est envoyée sur la céramique piezoélectrique d'un des lasers, après amplification et intégration. Le battement de fréquences est également détecté par un compteur de fréquences référencé à une horloge à rubidium. L'exactitude relative de cette référence est meilleure que $10^{-10}$ et cette valeur correspond à l'exactitude relative ultime de notre système.

\subsubsection{Détection de la phase du signal superhétérodyne}

L'expression des signaux de mesure et de référence est détaillée dans les références [20,23]. La phase synthétique $\Phi_{s}$ est donnée par :

$$
\Phi_{s}=\left[\Phi_{\text {probe }}\left(f_{2}\right)-\Phi_{\text {probe }}\left(f_{1}\right)\right]-\left[\Phi_{\text {ref }}\left(f_{2}\right)-\Phi_{\text {ref }}\left(f_{1}\right)\right]
$$

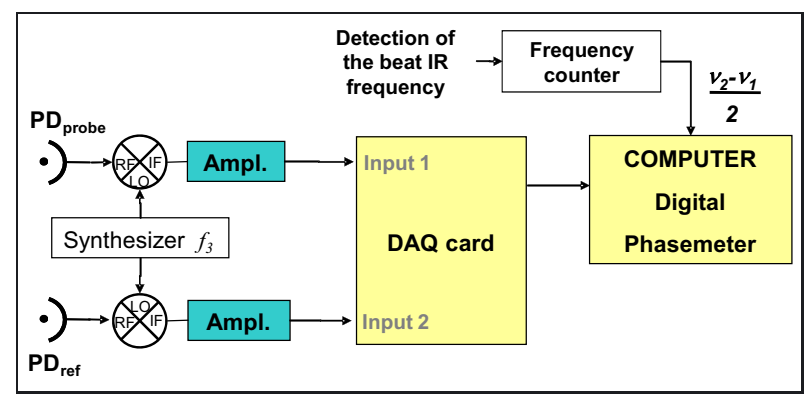

Fig. 5. - Système de détection superhétérodyne : AMPL, amplificateur ; $\mathrm{PD}_{\text {probe }}$, photodétecteur de mesure ; $\mathrm{PD}_{\text {ref }}$, photodétecteur de référence; IR, infrarouge; RF, radiofréquence; $\mathrm{LO}$, oscillateur local ; IF, intermediate frequency; DAQ, acquisition de données (5 Méch/sec, 12 bits).

où $\Phi_{\text {probe }}\left(f_{1}\right)$ (resp. $\left.\Phi_{\text {probe }}\left(f_{2}\right)\right)$ est la phase du signal de mesure à la fréquence $f_{1}$ (resp. $\left.f_{2}\right)$ et $\Phi_{\text {ref }}\left(f_{1}\right)$ (resp. $\left.\Phi_{\text {ref }}\left(f_{2}\right)\right)$ la phase du signal de référence à la fréquence $f_{1}$ (resp. $f_{2}$ ). La variation de la phase synthétique entre deux positions $z_{0}$ et $z$ du coin de cube est donnée par:

$$
\Phi_{s}(z)-\Phi_{s}\left(z_{0}\right)=\frac{4 \pi}{c}\left(n_{2} v_{2}-n_{1} v_{1}\right)\left(z-z_{0}\right)=\frac{4 \pi}{c} n_{g} \Delta v_{s} L .
$$

La distance $L=z-z_{0}$ entre ces deux positions peut donc être déduite de la variation de phase synthétique par la relation :

$$
L=\frac{\Phi_{s}(z)-\Phi_{s}\left(z_{0}\right)}{\frac{4 \pi}{c} n_{g} \Delta v_{s}} .
$$

La figure 5 illustre le système de détection superhétérodyne de la phase synthétique. Les signaux de mesure et de référence détectés sur les photodiodes $\mathrm{PD}_{\text {probe }}$ et $\mathrm{PD}_{\text {ref }}$ contiennent une composante à la fréquence $f_{1}$ et à la fréquence $f_{2}$. L'information sur la distance est contenue dans la phase à la fréquence $f_{2}-f_{1}$. Cette différence de fréquences est habituellement obtenue par élévation au carré des signaux contenant les fréquences $f_{1}$ et $f_{2}$, grâce à un mélangeur de fréquence ([19] par exemple). Cette technique a l'inconvénient d'utiliser un mélangeur de fréquences, pour élever au carré un signal envoyé sur les entrées IF et LO, qui convertit une variation d'amplitude du signal en variation de phase. Une variation d'amplitude du signal optique reçu est interprétée comme une variation de la distance mesurée et induit donc un biais sur la mesure. Pour éviter ce biais, nous avons choisi de ramener en bande de base les signaux de référence et de mesure par mélange de fréquence, avec un oscillateur à la fréquence $f_{3}$ pour obtenir des signaux aux fréquences $f_{2}-f_{3}(60 \mathrm{kHz})$ and $f_{1}-f_{3}(10 \mathrm{kHz})$. Après amplification, ces signaux sont convertis par une carte de conversion analogique/numérique et traités numériquement sous Labview. La phase superhétérodyne à $50 \mathrm{kHz}$ est extraite à partir des phases mesurées simultanément à $10 \mathrm{kHz}$ et $60 \mathrm{kHz}$.

\subsection{Résolution du système}

La résolution du système a été évaluée en réalisant une mesure de phase pendant environ $15 \mathrm{~h}$ dans une salle 


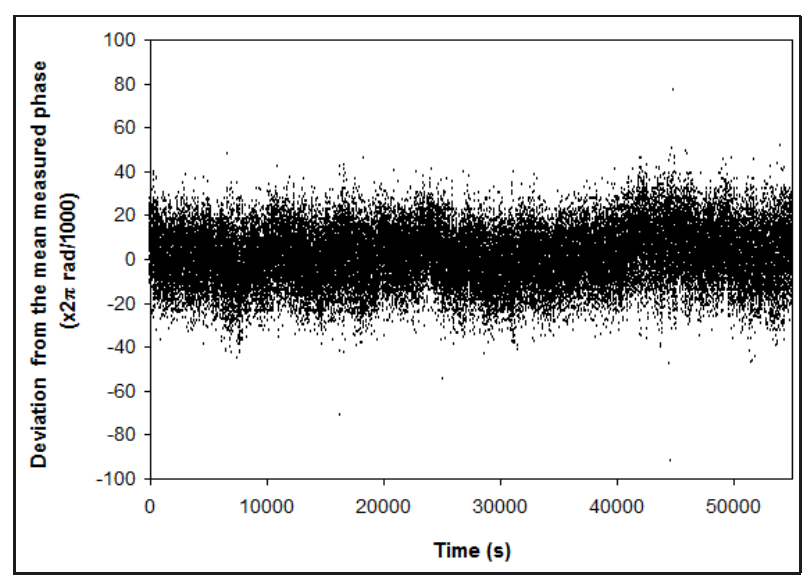

Fig. 6. - Caractérisation de la stabilité du système de mesure obtenue pour une longueur d'onde synthétique de $2,5 \mathrm{~cm}$; l'ordonnée est l'écart à la valeur moyenne de la phase mesurée exprimée en millième; le temps d'intégration pour chaque point est de $1 \mathrm{~s}$.

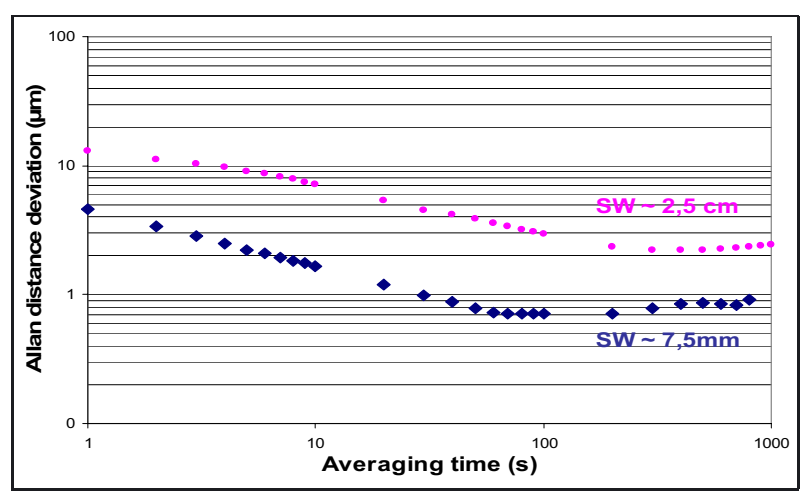

Fig. 7. - Écart type d'Allan (en micromètres) de la distance mesurée (environ 6,5 m) en fonction du temps d'intégration pour une longueur d'onde synthétique de l'ordre de $2,5 \mathrm{~cm}$ $\left(\Delta v_{s}=12 \mathrm{GHz}\right)$, ronds rouges, et de l'ordre de 7,5 $\mathrm{mm}\left(\Delta v_{s}=\right.$ $40 \mathrm{GHz})$, losanges bleus.

climatisée (fig. 6). La résolution obtenue pour un temps d'intégration de $1 \mathrm{~s}$ est d'environ un millième de la longueur d'onde synthétique. La figure 7 montre l'écart type d'Allan de la variation de distance mesurée (autour d'une distance nominale de 6,5 m) en fonction du temps d'intégration pour une longueur d'onde synthétique de 7,5 $\mathrm{mm}$ (losanges bleus) et de $2,5 \mathrm{~cm}$ (ronds rouges). Les deux courbes montrent un plateau qui indique le temps d'intégration optimal pour obtenir la meilleure résolution. Pour une longueur d'onde synthétique de 7,5 mm (écart de fréquence de $40 \mathrm{GHz}$ entre les deux lasers) la meilleure résolution obtenue est de $700 \mathrm{~nm}$ pour $60 \mathrm{~s}$ d'intégration.

\section{Comparaison en intérieur}

Nous avons validé le dispositif par comparaison à un système de référence. Ce système est le banc de déplacement de $50 \mathrm{~m}$ du VSL (Van Swinden Laboratory, Delft, Laboratoire national de métrologie hollandais) où nous avons transporté le système du LCM. Le banc est contrôlé par un interféromètre à comptage de franges

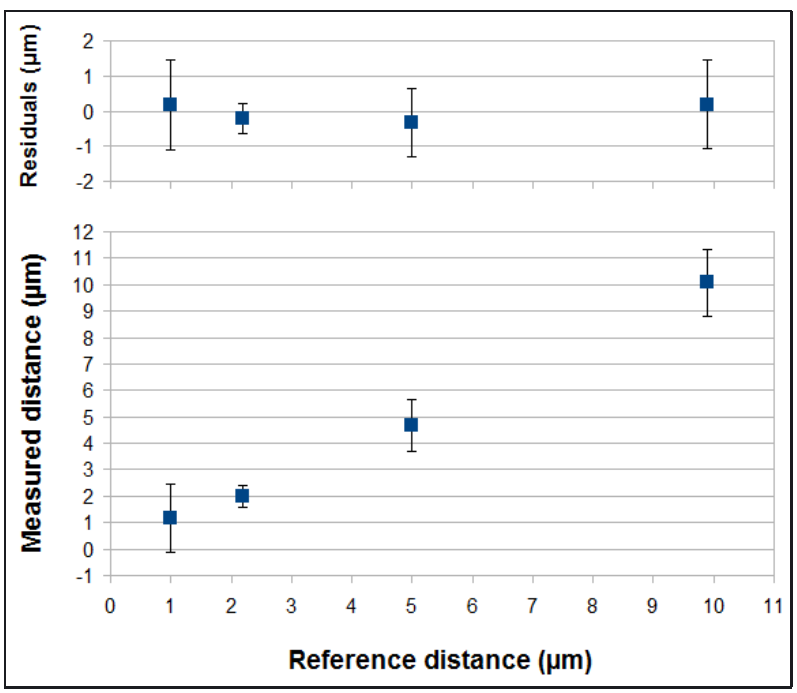

Fig. 8. - Distance mesurée en fonction d'une distance de référence. Les valeurs moyennes de six mesures sont représentées avec les écarts types d'échantillons correspondants.

(Spindler \& Hoyer, He-Ne laser) dans une pièce contrôlée en température.

\subsection{Résolution}

Nous avons dans un premier temps vérifié la résolution de l'ADM. Un coin de cube a été fixé sur une platine de déplacement micrométrique et déplacé sur une très faible course de $10 \mu \mathrm{m}$. Le déplacement a été mesuré avec l'ADM et l'interféromètre de référence. Les écarts entre la valeur mesurée et la valeur de référence sont représentés sur la figure 8 . On voit clairement qu'un déplacement de $1 \mu \mathrm{m}$ est détecté par l'ADM, ce qui confirme la résolution meilleure que $1 \mu \mathrm{m}$ (soit mieux que 1/7 500 de la longueur d'onde synthétique). L'écart de fréquence entre les deux lasers était de $40 \mathrm{GHz}$.

\subsection{Exactitude}

Nous avons ensuite caractérisé la justesse de l'instrument du LCM. Les mesures se sont déroulées en plusieurs étapes :

(1) une position zéro du coin de cube (CC) est mesurée à un point $\mathrm{A}\left(z_{A}\right)$ du banc en utilisant notre $\mathrm{ADM}$;

(2) le coin de cube est déplacé du point $\mathrm{A}$ à un point $\mathrm{B}$ du banc pendant que le déplacement est mesuré par l'interféromètre de référence;

(3) la distance absolue en $\mathrm{B}\left(z_{B}\right)$ est mesurée par notre $\mathrm{ADM}$;

(4) La distance entre les points $\mathrm{A}$ et $\mathrm{B}\left(z_{B}-z_{A}\right)$ est déduite des mesures en $\mathrm{A}$ et en $\mathrm{B}$ et comparée à la valeur donnée par l'interféromètre de référence. Les distances ont été mesurées en utilisant une fréquence synthétique de $40 \mathrm{GHz}$ et $10 \mathrm{~s}$ de temps d'intégration, donnant une résolution de $2 \mu \mathrm{m}$ à $3 \mu \mathrm{m}$. 


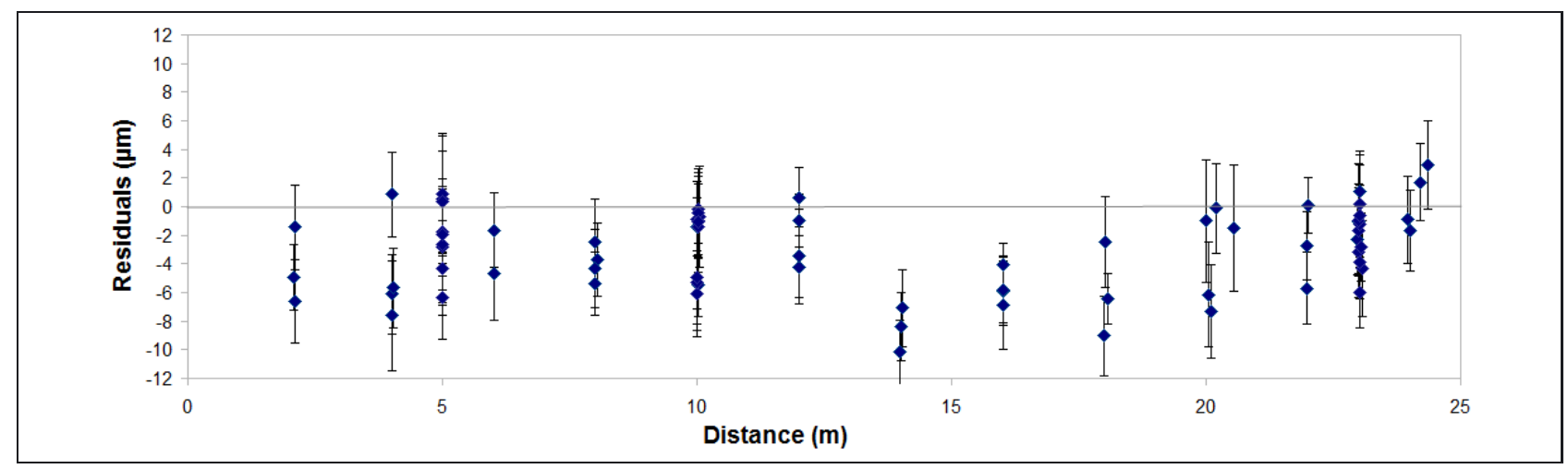

Fig. 9. - Comparaison en intérieur : Résidus (en $\mu \mathrm{m}$ ) entre la distance mesurée par l'ADM du LCM et la valeur donnée par un interféromètre de référence.

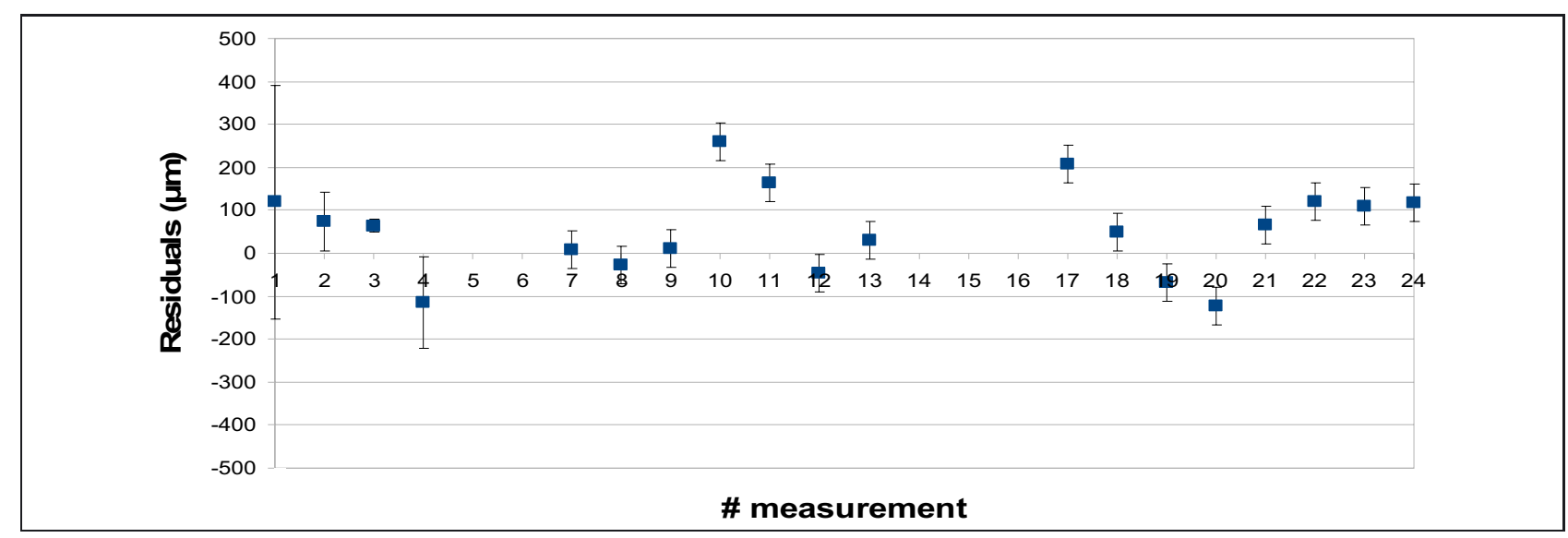

Fig. 10. - Écarts («residuals» sur la figure) entre la valeur vraie et les valeurs mesurées pour le pilier 1 de la ligne géodésique de référence de Nummela. Les barres d'incertitude correspondent à l'écart type de mesure.

Les mesures ont été réalisées en faisant plusieurs allers et retours du coin de cube le long du banc. Les mesures ont été répétées plusieurs fois et sont résumées sur la figure 9. Nous n'observons pas d'augmentation de l'erreur avec la distance mesurée. La figure 9 montre néanmoins un léger biais $m_{\text {res }}=4 \mu \mathrm{m}$ (l'écart moyen par rapport à la valeur vraie). L'écart type des écarts entre la valeur vraie et la valeur mesurée pour chaque distance considérée sur la figure 9 vaut $\sigma_{\text {res }}=3 \mu \mathrm{m}$. Nous estimons donc l'incertitude type de la valeur donnée par l'ADM à $\sqrt{m_{\text {res }}^{2}+\sigma_{\text {res }}^{2}}=5 \mu \mathrm{m}$. En considérant un facteur d'élargissement de 2, l'exactitude du système ADM du LCM est donc estimée à $10 \mu \mathrm{m}$.

\section{Comparaisons en extérieur}

Les comparaisons en extérieur ont été réalisées sur une ligne géodésique du Finnish Geodetic Institute (FGI, Nummela, Finlande). Cette ligne géodésique est le système de référence idéal et le mieux connu au monde puisque sa traçabilité est assurée depuis 70 ans et montre une stabilité extrêmement bonne [24]. Cette ligne est constituée de six piliers d'observation à $0,24 \mathrm{~m}, 72 \mathrm{~m}$, $216 \mathrm{~m}, 432 \mathrm{~m}$ et $864 \mathrm{~m}$, dont les écartements sont traçables au mètre par une technique d'interférométrie en lumière blanche réalisée par un interféromètre de Väisälä, avec des incertitudes variant de $22 \mu \mathrm{m}$ à $75 \mu \mathrm{m}$ [6]. Cette technique consiste à comparer la longueur d'un cylindre étalon en quartz de $1 \mathrm{~m}$ aux différents écartements entre les piliers. Il faut noter que ce type d'étalonnage nécessite des compétences très spécifiques que la mesure des cinq piliers peut durer 2 mois. D'autre part, les mesures avec cet interféromètre ne peuvent se faire que lorsque les conditions climatiques sont très favorables : le gradient de température tout le long de la ligne doit être inférieur à $0,1^{\circ} \mathrm{C}$, le taux d'humidité doit être homogène à mieux que $4 \%$ et le vent doit être suffisamment faible pour obtenir des signaux mesurables. Elles doivent en outre se faire de nuit compte tenu du très faible flux de la source de lumière blanche utilisée.

Chaque mesure a consisté à (1) mesurer la distance $D_{0}$ entre la tête de mesure et le pilier 0 ; (2) mesurer la distance $D_{i}$ entre la tête de mesure et le pilier $i$ ( $i=\{1: 5\}) ;(3)$ en déduire la distance $D_{i}-D_{0}$. Néanmoins pour chaque pilier, la mesure de l'ordre d'interférence qui consiste à faire la mesure pour plusieurs fréquences synthétiques n'a été réalisée qu'une seule fois par pilier. La tête de mesure a été placée sur un pilier dédié (pilier -1), pointant dans la direction du coin de cube fixé sur le pilier à mesurer sur un support approprié (fig. 10). Les mesures ont été réalisées de cette façon pour les piliers de $24 \mathrm{~m}$ (pilier 1) à 864 m (pilier 5). 


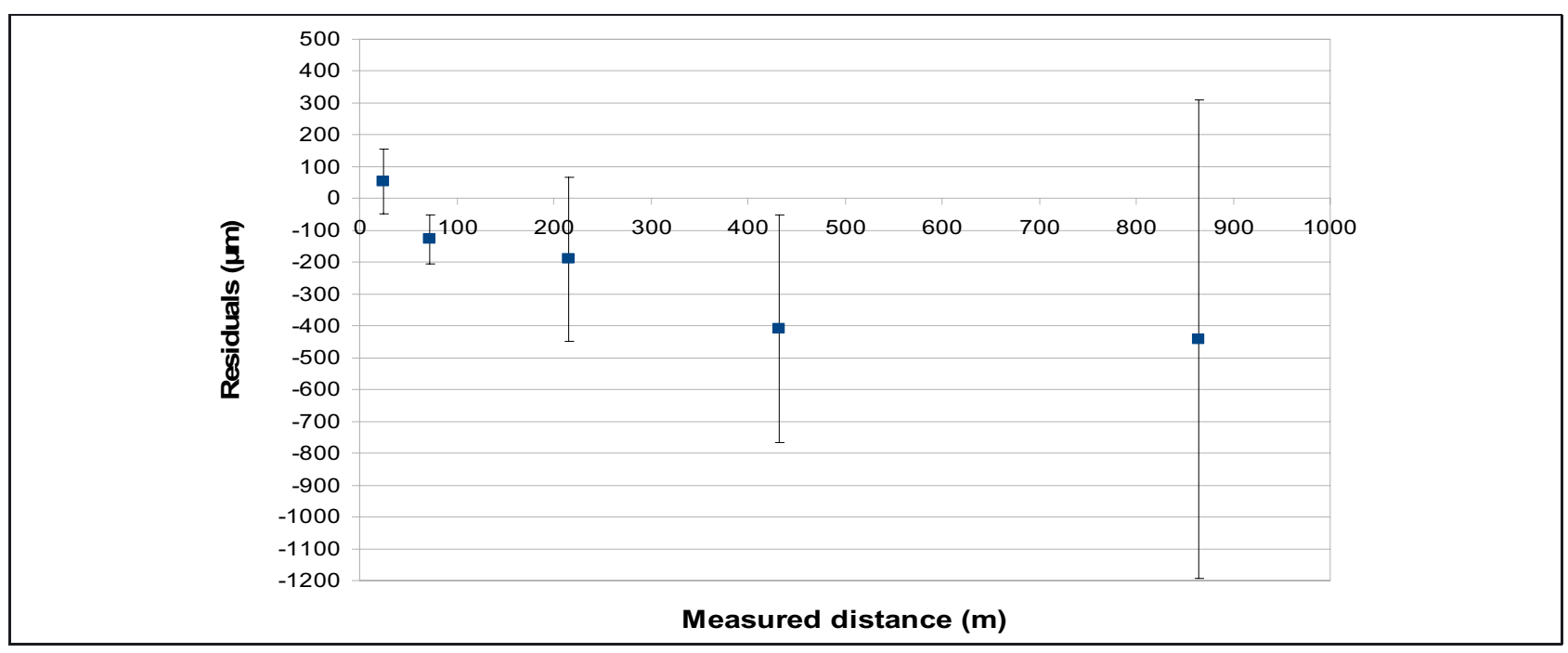

Fig. 11. - Écarts entre la valeur vraie et les valeurs mesurées pour les piliers 1 à 5 de la ligne géodésique de référence de Nummela ( $24 \mathrm{~m}, 72 \mathrm{~m}, 216 \mathrm{~m}, 432 \mathrm{~m}$ et $864 \mathrm{~m})$.

Lors de cette campagne de mesure qui a précédé les mesures en intérieur développées ci-dessus, la fréquence synthétique était de $12 \mathrm{GHz}$, jusque-là limitée par la bande passante du détecteur de battement infrarouge des lasers YAG.

La figure 11 montre les résultats de 24 mesures réalisées entre les piliers 0 et 1 , séparées de $24 \mathrm{~m}$. Pour ce pilier, les mesures ont été effectuées sur 3 jours. Chaque point de mesure a été réalisé après avoir retiré puis réinstallé le coin de cube sur son support. L'ordonnée représente l'écart entre la valeur mesurée et la valeur vraie, connue du fait des mesures régulières de la ligne géodésique. L'écart moyen à la valeur vraie est de $53 \mu \mathrm{m}$, avec une incertitude type d'échantillon d'environ $100 \mu \mathrm{m}$.

Les mesures ont ensuite été réalisées pour les quatre autres piliers, jusqu'à $864 \mathrm{~m}$ et sont reportées sur la figure 12. Les barres d'incertitude correspondent à la dispersion de plusieurs mesures réalisées sur plusieurs jours. Jusqu'à $216 \mathrm{~m}$, une mesure individuelle de 50 points de $1 \mathrm{~s}$ peut présenter un écart type de $30 \mu \mathrm{m}$ si les conditions atmosphériques sont favorables pendant la mesure. Audelà du pilier à $216 \mathrm{~m}$, la plus faible dispersion sur une série de mesures de $50 \mathrm{~s}$ était de $75 \mu \mathrm{m}$. La dispersion importante des mesures (écart de $700 \mu \mathrm{m}$ pour le dernier pilier à $864 \mathrm{~m}$ ) s'explique par l'incertitude liée à l'évaluation des paramètres atmosphériques pour la détermination de l'indice de groupe. La pression atmosphérique et le taux d'humidité ont été mesurés par une station météorologique étalonnée, fournie par le FGI. L'incertitude liée à la mesure d'humidité amène une contribution d'environ $20 \mu \mathrm{m} / \mathrm{km}$ et celle liée à la mesure de la pression atmosphérique de $60 \mu \mathrm{m} / \mathrm{km}$. La température de l'air est mesurée par un psychromètre de type Assmann placé à proximité du pilier de mesure, à l'abri su soleil. Pour des raisons pratiques, la température n'était mesurée qu'en un point de la ligne (à proximité du coin de cube). Il est difficile d'estimer dans ces conditions l'incertitude liée à la mesure de la température effective le long du chemin

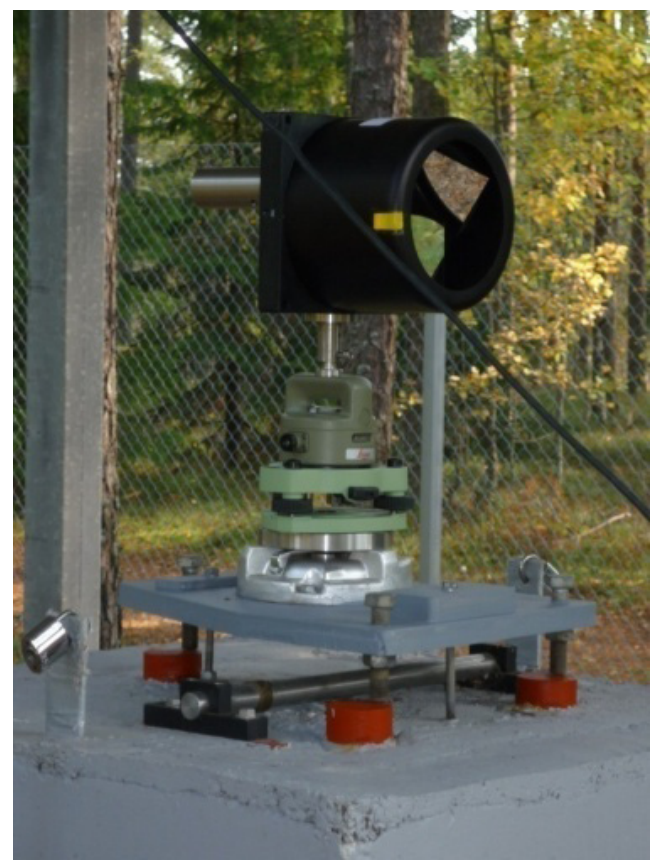

Fig. 12. - Support type et coin de cube pour les mesures de distance entre piliers.

de mesure. Néanmoins, une valeur de $0,5{ }^{\circ} \mathrm{C}$ à $1{ }^{\circ} \mathrm{C}$ pour cette incertitude n'est pas déraisonnable. L'incertitude liée à la température se propage sur l'incertitude de distance avec un coefficient de $1 \mathrm{~mm} /{ }^{\circ} \mathrm{C} / \mathrm{km}$. Une incertitude type de l'ordre du millimètre liée à la détermination de l'indice de l'air est compatible avec la dispersion des mesures de l'ordre de $700 \mu \mathrm{m}$ observées sur le pilier 5 (fig. 12).

\section{Conclusion et perspectives}

Nous avons réalisé un télémètre transportable fondé sur l'interférométrie à longueur d'onde synthétique. 
Cet instrument a démontré une résolution meilleure que $1 \mu \mathrm{m}$ sur une distance allant jusqu'à $25 \mathrm{~m}$ en intérieur. Nous avons démontré sa robustesse par des mesures en extérieur jusqu'à 864 m dont l'exactitude était limitée par la mesure des paramètres atmosphériques.

L'intérêt essentiel de la détection superhétérodyne mise en place est de pouvoir bénéficier d'une fréquence synthétique élevée (40 GHz), et donc d'une bonne résolution de distance, en détectant la phase de ce signal sur des signaux optiques de basses fréquences de quelques dizaines de mégahertz (la fréquence de décalage des modulateurs acousto-optiques). Ceci permet de s'affranchir des difficultés liées au traitement de signaux hyperfréquences.

La limitation essentielle, comme attendue, vient de la prise en compte des paramètres atmosphériques pour la détermination de la vitesse de propagation de la lumière le long du chemin à mesurer. Des solutions sur la base de la connaissance de la dispersion chromatique de l'indice de l'air ont déjà été proposées dans le passé [25] et permettent de s'affranchir de la mesure de température et de la pression atmosphérique pour mesurer une distance par voie optique. Ce principe implique la mesure de la distance à deux longueurs d'onde très éloignées $(532 \mathrm{~nm}$ et $1064 \mathrm{~nm}$ par exemple). La modification du système actuel pour utiliser ce principe de compensation partielle d'indice ne nous semble pas adaptée à un système transportable et utilisable par des utilisateurs non spécialistes. Pour cette raison, nous sommes engagés dans la réalisation d'un nouveau système de télémétrie avec compensation partielle d'indice de l'air fondé sur des diodes lasers. Ce travail s'inscrit dans les projets collaboratifs de l'Euramet/EMRP (JRP) SURVEYING [26] et LUMINAR [27].

\section{Remerciements}

Le projet collaboratif «Absolute Long-distance Measurement in Air 》 est un projet de l'EMRP (European Metrology Research Program) d'Euramet. Ce travail a été en partie financé par le progamme ERANETPlus de la communauté européenne sous le numéro d'agrément 217257.

\section{Références}

[1] Méchain M. et Delambre M., «Base du système métrique décimal ou mesure de l'arc du méridien compris entre les parallèles de Dunkerque et Barcelone, exécutée en 1792 et années suivantes », Suite des Mémoires de l'institut, Tome Premier, Paris, 1806.

[2] Colosimo G., Crespi M., Mazzoni A., Jones M. et MISSIAEN D., "Determination of the CNGS global geodesy OPERA public note", 132, 2011, 1-7.

[3] Jokela J., Häkli P., Poutanen M., Kallio U. et AHOLA J., "Improving Length and Scale Traceability in Local Geodynamical Measurements. In: Geodesy for Planet Earth", Proceedings of the 2009 IAG Symposium, Buenos Aires, Argentina, Eds. Kenyon S., Pacino M., Marti U., International Association of Geodesy Symposia, 136, Springer Verlag., 59-66, 2012, DOI : 10 . 1007/978-3-642-20338-1_8.
[4] http://www.leica-geosystems.com/downloads123/zz/tps/ nova_tm50/brochures-datasheet/Leica_Nova_TM50 DAT_fr.pdf (consulté le 12 janvier 2015).

[5] ISO, "Optics and optical instruments - Field procedures for testing geodetic and surveying instruments Part 4: Electro-optical distance meters (EDM instruments)", ISO 17123-4, 2012.

[6] JoKela J. et HÄKLI P., "Interference measurements of the Nummela standard baseline in 2005 and 2007”, Suomen geodeettinen laitos, 144, 2010, 1-85.

[7] Pollinger F., Meyer T., Beyer J., Doloca N. R., SCHEllin W., NiEMEIER W., JOKEla J., HAKLI P., ABou-ZeID A. et MeINERs-HAGEN K., "The upgraded PTB 600 m baseline: a high-accuracy reference for the calibration and the development of long distance measurement devices", Meas. Sci. Technol., 23, 2012.

[8] Proceedings of the workshop on the use and calibration of the Kern ME5000 Mekometer, Stanford linear accelerator center, 18-19 juin 1992, http://www.slac.stanford.edu/ pubs/slacreports/reports03/slac-r-403.pdf (consulté le 12 janvier 2015).

[9] Bobroff N., "Recent advances in displacement measuring interferometry", Meas. Sci. Technol., 4, 1993, 907-926.

[10] Polhemus C., "Two-wavelength interferometry", Appl. Opt., 12, 1973, 2071-2074.

[11] Gelmini E., Minoni U. et Docchio F., "A tunable, double-wavelength heterodyne detection interferometer with frequency-locked diode-pumped Nd:YAG sources for absolute measurements", Rev. Sci. Instrum., 66, 1995, 4073.

[12] DE GRoOT P. et KISHNER S., "Synthetic wavelength stabilization for two-color laser-diode interferometry", Appl. Opt., 30, 1991, 4026.

[13] Schuhler N., SalvadÉ Y., LÉvêQue S., DÄNDliker R. et HOLZWARTH R., "Frequency-comb-referenced twowavelength source for absolute distance measurement", Opt. Lett., 31, 2006, 3101.

[14] Salvadé Y., Schuhler N., LÉvêQue S. et Le Floch S., "High-accuracy absolute distance measurement using frequency comb referenced multiwavelength source", Appl. Opt., 47, 2008, 2715 .

[15] Mahal V. et ARIE A., "Distance measurements using two frequency stabilized Nd:YAG lasers", Appl. Opt., 35, 1996, 3010-3015.

[16] BECHSTEIN K.-H. et FUCHS W., "Absolute interferometric distance measurements applying a variable synthetic wavelength", J. Opt., 29, 3, 1998, 179-182.

[17] CIDDOR P.E., "Refractive index of air: New equations for the visible and near infrared", Appl. Opt., 35, 1996, 1566-1573.

[18] Ciddor P.E. et HILl R.J., "Refractive index of air. 2. Group index", Appl. Opt., 38, 1999, 1663-1667.

[19] Dandliker R., Thalmann R. et Prongué D., “Twowavelength laser interferometry using superheterodyne detection", Opt. Lett., 13, 1998, 339-341. 
[20] Azouigui S., BAdR T., WALlerand J.-P., Himbert M., SALGADO J.-A., SENELAER J.-P., KWASNIK F. et JUNCAR P., "Transportable Distance Measurement System for Long-Range Applications", IEEE Trans. Instrum. Meas., 60, 2011.

[21] Michelson A., «Détermination expérimentale de la valeur du mètre en longueurs d'ondes lumineuses » (1894), https://archive.org/details/dterminationex00mich (consulté le 12 janvier 2015).

[22] SALgADo J.A., «contrôle et étalonnage des cales étalons », Techniques de l'ingénieur, Base documentaire Mesures de longueurs et d'angles, R1245 v2, 2008.

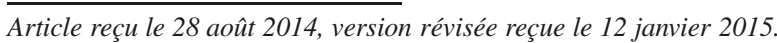

[23] Azouigui S., Badr T., Wallerand J.-P., Himbert M., SAlgado J.-A. et JUNCAR P., "Transportable distance measurement system based on superheterodyne interferometry using two phase-locked frequency-doubled Nd:YAG lasers", Rev. Sci. Instrum., 81, 2010, 053112.

[24] JoKelA J. et HäKLI P., "Current research and development at the Nummela standard baseline", XXIII FIG Congress "Shaping the Change", Munich, Allemagne, 8-13 oct. 2006, http://www.fig.net/pub/fig2006/papers/ ps05_02/ps05_02_02_jokela_hakli_0371.pdf (consulté le 12 janvier 2015).

[25] EARNSHAW K. et OWENS J., "A dual wavelength optical distance measuring instrument which corrects for air density", IEEE J. Quantum Electron., 3, 1967, 544-550.

[26] http://www.ptb.de/emrp/surveying.html (consulté le 12 janvier 2015).

[27] http://projects.npl.co.uk/luminar/ (consulté le 12 janvier 2015). 